\title{
Rethinking life and predicting its origin
}

\author{
Diogo Gonçalves ${ }^{1 *}$ \\ ${ }^{1}$ Instituto Superior Técnico, University of Lisbon; Lisbon, 1049-001, Portugal \\ * diogo.v.goncalves@ tecnico.ulisboa.pt
}

The definition, origin and recreation of life remain elusive. As others have suggested, only once we put life into reductionist physical terms will we be able to solve those questions. To that end, this work proposes the phenomenon of life to be the product of two dissipative mechanisms. From them, one reinterprets extant biological life and deduces a testable scenario for its origin. The proposed theory of life allows its replication, reinterprets ecological evolution, brings new constraints to astrobiology and lays the foundations for groundbreaking technologies.

\section{Introduction}

The pursuit of the essence of life has compelled a large and multi-disciplinary community of researchers. They have been trying to create life de novo $(1,2)$, to address its biological origin (3), to understand its metabolic composition (4-6), to develop undisputed biosignatures $(7,8)$ or to define it $(9-12)$. In the latter case, researchers have created operational definitions of life by stating the properties we would expect from a living system based on what is known from biology $(9,12)$. As Cleland and Chyba put it, the standard approach has been equivalent to defining water unaware of what molecules are (12). We have listed the properties of life and expected that their overlap defines only one phenomenon. That approach has made us preconceive reduced carbon species $(9)$, cells $(9,13)$, genetic polymers $(13,14)$, semipermeable membranes (15) and Darwinian evolution (16) as characteristics of life based on their omnipresence in biological organisms. Inevitably, one can find inconsistencies when considering each of those properties as pillars of life $(11,12,16,17)$.

Without a simple explanation for the phenomenon of life, those trying to recreate the origin of biological life have pursued divergent "worlds" and theories (18-21). On one side there have been metabolism-first (22) advocates which attempt to reproduce extant biological metabolic pathways, particularly autocatalytic ones (23). Among the biggest obstacles to this approach has been the lack of catalytic specificity (24). Researchers of genetics-first theories thus argue that, before anything else, there must have been selfreplicating information-carrying catalysts to structure the metabolism. Starting from RNA (25) and RNAlike (14) structures, they have aimed at creating self-replicating catalysts that make an ordered metabolism 
possible. But the difficulty to create those structures has prevented the wide acceptance of this alternative approach.

In that context, some argue that physics is due to resolve the standing problems about life and its origin by explaining this phenomenon under a universal theory of life (26). This work embodies that argument. Through two simple dissipative mechanisms (herein the "principles of life"), it explains life starting from the $2^{\text {nd }}$ Law of Thermodynamics. Using those principles, the origin of life as we know it is then deduced $a b$ initio.

\section{The principles of life}

The production of entropy. The $2^{\text {nd }}$ Law of Thermodynamics states that all processes in nature increase the entropy of the Universe. Depending on the disequilibrium being dissipated, there may be different entropyproducing processes: heat flows, mechanical expansions, or chemical reactions and diffusion processes. When there are no thermodynamic disequilibria, a system has achieved maximum entropy, no further directionality exists, and the system becomes immutable.

Life is a chemical phenomenon, i.e., it exists in the realm of disequilibria on chemical compositions. The processes by which those disequilibria dissipate (e.g., diffusions and chemical reactions) usually occur at constant pressure and temperature. In those conditions, we can assess spontaneity (i.e., compliance with the $2^{\text {nd }}$ Law of Thermodynamics) by ensuring negative variations on Gibbs free energy. Albeit the formulations are interchangeable, I will refer only to the production of the Universe's entropy as the responsible for the spontaneity of any (chemical) process.

$\boldsymbol{I}^{\text {st }}$ Principle. The first proposed principle of life addresses life's capacity to "build". It regards its ability to organise matter into out-of-equilibrium compositions, which then amount to complex biomolecules, organisms and entire forests. It regards its ability to undergo the processes that progressively build those thermodynamic disequilibria. In other words, the $1^{\text {st }}$ Principle notes life's inherent ability to create chemical disequilibria.

The creation of chemical disequilibria supposes the existence of "entropy-destroying" (27) processes such as endergonic chemical reactions or diffusions against concentration gradients, in apparent violation of the $2^{\text {nd }}$ Law. As entropy is constantly being produced, the creation of chemical disequilibria must be coupled $(28,29)$ to the dissipation of other (higher) disequilibria. In biological life, active transport and endergonic (entropy-destroying) reactions only take place when coupled to exergonic (entropy-producing) ones or to diffusions along concentration gradients. Other natural instances of creation of chemical disequilibria include the excitation of electrons or endergonic photochemistry performed at the expense of the dispersal of sunlight's energy. That is how the Universe is always producing entropy and yet creates chemical disequilibria. 
In a finer description, the creation of a disequilibrium results from a sequence of entropy-producing steps, where a fraction of the dissipating disequilibrium gets preserved in a different form. As entropy and free energy are state variables, we can then interpret such a sequence as a coupling between one entropyproducing process and an entropy-destroying one. But in reality, there is a constant production of entropy where part of a disequilibrium is converted (29) into another form. In the phenomenon of life, disequilibria are converted into chemical disequilibria; where a particle (e.g., electron, atom or molecule) is forced outof-equilibrium, and later tends to diffuse or react towards maximum entropy.

For simplicity, I will call building system to the process of creation of chemical disequilibria and all the processes by which they dissipate.

$2^{\text {nd }}$ Principle. Whereas the $1^{\text {st }}$ Principle of life regards the creation of chemical disequilibria, the second proposed principle addresses their dissipation.

Most of the times, chemical disequilibria dissipate according to environmental constraints, with no memory about past dissipations. In such cases, environmental constraints alone guide the production of entropy - its direction and rate - by determining which chemical compositions are the most stable and kinetically accessible. When the environment does not change, neither do those constraints. The dissipative routes may be perpetually predictable (e.g., on a battery) or perpetually random (e.g., on Miller experiments (30,31)). But anyhow, the constraints exerted on them do not change (Fig. 1A).

Sometimes, the dissipation of the disequilibria is influenced not only by the environment but also by previous dissipative processes: the dissipation of the disequilibria changes the thermodynamic and/or kinetic constraints exerted on itself (Fig. 1B). Examples of it include a river that shapes the riverbed it flows on, a brain whose activity updates its neural pathways (forming memories), or an oscillating chemical reaction (32). The production of entropy is constrained not solely by the environment, but also by its history. We may say that the production of entropy has a memory effect, that it can update its constraints, or that it is self-constrained. That is the second, and last, axiom of life.

Biological metabolisms have become an extreme expression of this $2^{\text {nd }}$ Principle. After the dissipation of environmental disequilibria has created chemical ones ( $1{ }^{\text {st }}$ Principle $)$, the latter dissipate through metabolic reactions and diffusion processes largely constrained by their peptidic and nucleotidic products. Without self-constraints on the dissipation of their chemical disequilibria, living systems would not sustain homochirality in their components, would not differentiate into numerous cell types, and - as will be explored - would not become self-replicating.

Taken together, the creation of chemical disequilibria ( $1^{\text {st }}$ Principle) and their self-constrained dissipation ( $2^{\text {nd }}$ Principle) are the mechanisms encoded by "life". From them, I will reinterpret the composition of life as we know it and reconstruct its properties. The fact that biological life was always the product of those 
principles creates powerful constraints on its origin. In the last section, I use the proposed principles and the extant forms of biological life to predict its origin through a testable scenario.

A

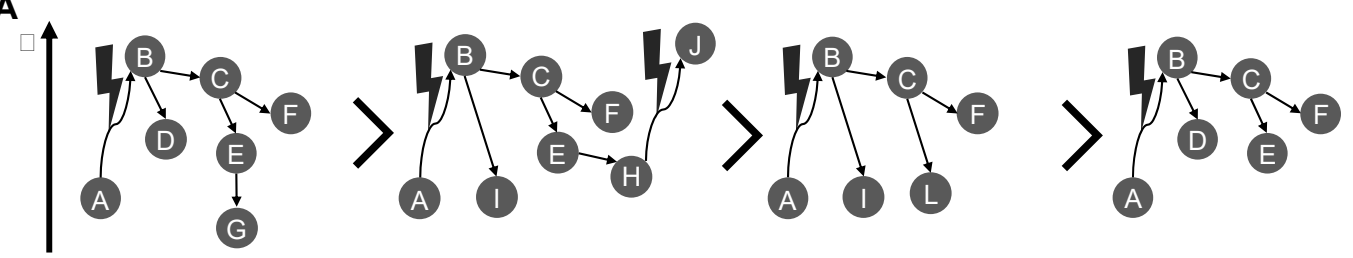

B

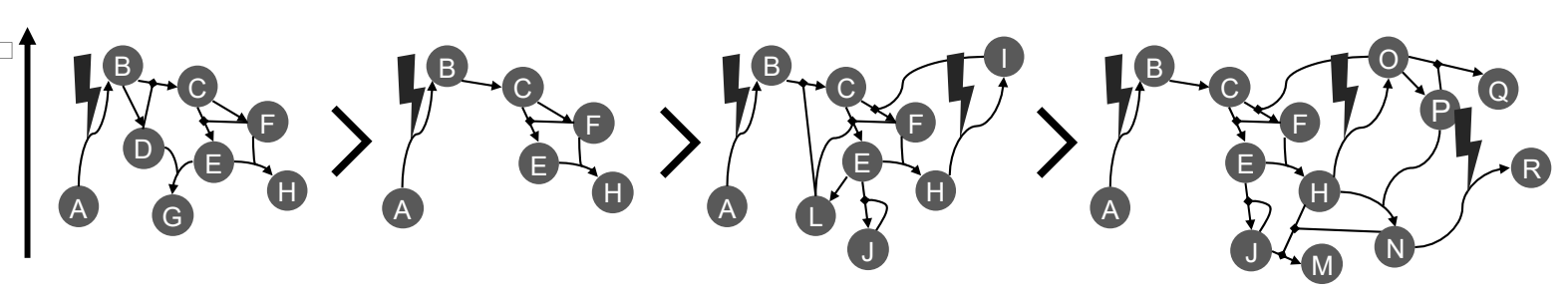

Fig. 1. Building systems over time. An entropy-destroying process - e.g., the formation of compound B from compound A - is only possible when coupled to an entropy-producing one (lightning). The creation of chemical disequilibria precedes their dissipation, which may take place through chemical reactions (arrows) towards species of ever lower chemical potential $(\mu)$. A. When an unchanging environment fully determines the dissipative processes (i.e., the chemical reactions), they perpetually occur under the same constraints. B. When previous versions of the dissipative processes also constrain the dissipation, the degrees-of-freedom may then change over time and a living system emerges.

Living systems. A living system is the persistent creation of a chemical disequilibrium and its selfconstrained dissipation (Fig. 1B). A system is alive as long as chemical disequilibria is created, and the subsequent production of entropy is self-influenced. The composition of a living system includes the constructs that create the chemical disequilibrium (by coupling it to the dissipation of another disequilibrium), and the products of the interacting dissipative processes.

Whereas extant biological autotrophs create their chemical disequilibria - as out-of-equilibrium electrons from sunlight or environmental redox gradients, heterotrophs create their chemical disequilibria - as chemiosmotic gradients and out-of-equilibrium phosphorylated metabolites - from the (chemical) disequilibria built by autotrophs. Those created disequilibria then dissipate by reducing and phosphorylating other molecules, followed by a chain of other dissipative processes including varied chemical reactions, and the formation of chemiosmotic gradients and macromolecular conformational tensions. As said, all those processes are heavily constrained by their products, e.g., by enzymes, polynucleotides or interactions and reactions among metabolites. Thus, all biological organisms are, necessarily, living systems. 
At the beginning of life, there was a simple living metabolism (e.g., Fig. 1B). Then, it was multiplied into several ones which, at some point, would interact among themselves, and so constrain each other's formation and/or composition. So were born communities. Communities are living systems whose selfconstraining composition includes other living systems (Fig. 2). They are entirely analogous to (and extensions of) their constituent metabolisms.
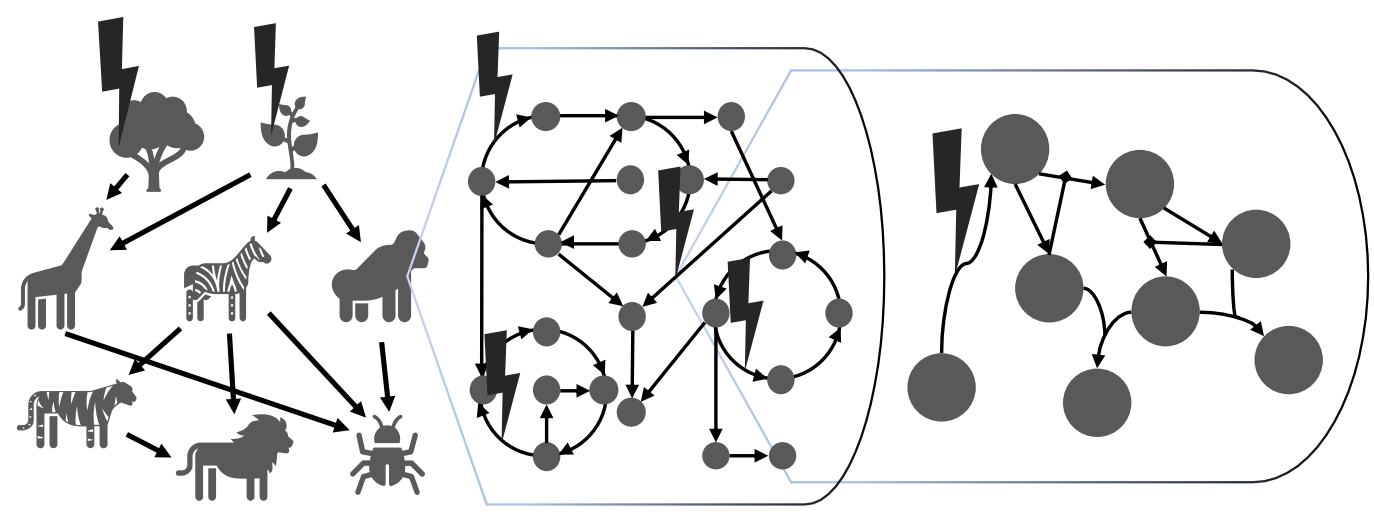

Fig. 2. The phenomenon of life at different scales. Life may expand from living metabolisms (centre) to living communities (left) and smaller metabolic living systems (right, see "The exploration of entropic dams"). Ubiquitous to all scales is the creation of chemical disequilibria and mechanisms by which their dissipation constrains itself.

Different living scales combine different self-constraining mechanisms.

\section{The composition of life}

Rethinking biological life as an expression of the two proposed principles answers long-standing questions about its composition. Before proceeding with this discussion, it is profitable to define selection and evolution in living systems in consonance with those principles.

Selection. In the present context, selection can be viewed as the formation of each component of a living system. In a more general formulation, selection is the undertaking of each dissipative process of a living system. In a living metabolism, it regards the formation of each metabolite, concentration gradient, or any other metabolic component. In a community, it also considers the formation and maintenance of its living components.

Evolution. Along the same line, a living system evolves when the constraints on its dissipation change. For instance, a biological metabolism evolves when a genetic mutation creates new constraints on the selection of proteins; and a biological community evolves upon seasonal changes in environmental constraints. The $2^{\text {nd }}$ Principle (a self-constrained dissipation) is the postulate that enables the evolution of a living system, even in unchanged environments (Fig. 1B).

Self-replicating compositions. It is well-known that, at the beginning of an ecological succession, a community has a simple network of living systems. Its composition (commonly a population of lichens) 
imposes few self-constraints, which actually favour the selection of new organisms. But as time goes by, that living community will expand its dissipative processes and then stabilise in a climax composition (for instance, a rainforest). In this climax stage, the living community heavily constrains itself and creates the conditions for the sole selection of its components. It becomes self-replicating and attains a stable composition.

Crucially, the same happens to metabolisms. At its origin, the biological metabolism had a simple pioneer composition. As it is always adapting to itself ( $2^{\text {nd }}$ Principle), self-favouring portions became, serendipitously, recurrent. With time, the evolution of the biological metabolism has slowed down, as it stabilised in a composition which favours its sole selection. It has become self-replicating - the stable state of any self-constraining dissipative system. It is in this later stage of evolution that we find all biological metabolisms. As explored in the next section, this biased observation has led to the widespread (33), yet misleading, association of life with self-replication.

Evolutive patterns. The $2^{\text {nd }}$ Principle may exist by a myriad of mechanisms. In living metabolisms, the selfconstraining mechanisms come down to molecular interactions. In living communities, they also include numerous interactions between its living systems (34). In this sub-section, I show how those mechanisms may yield some predictable patterns in the composition of a living system.

By and large, the chemical disequilibria of biological metabolisms dissipate through chemical reactions, which are constrained kinetically and thermodynamically. Thermodynamic constraints regard the topography of the thermodynamic landscape - i.e., the chemical potential of the accessible products. According to the $2^{\text {nd }}$ Principle, that thermodynamic landscape will adapt to new dissipative processes. That is the case when a new metabolite is formed that stabilises, reacts, or interacts with other metabolites. The latter's effective concentrations lower, and so do their chemical potentials. The new thermodynamic landscape will then favour their future selection (formation) as they become a more favourable route for the dissipation of chemical disequilibria.

In the aftermath of the long $(35,36)$ evolutionary succession of biological metabolisms, we could expect some signs of such thermodynamic self-constraints. And indeed, biological metabolites are not only more hydrophilic and thermodynamically stable than abiotic molecules, but they are also optimised to interact among themselves (37). As reactions and interactions between two metabolites stabilise them and their precursors - making their future selection more favourable -, the composition of any living metabolism will tend to interactomes like biological cells' (38).

In a different realm, the maintenance of symmetry-breaking properties, such as homochirality, is an example of the patterns we can predict to occur in living metabolisms, thanks to kinetic self-constraints on chemical reactions. 
Entropic dams. After water accumulates gravitational potential on mountain reservoirs, it flows downhill by whatever accessible means. Analogously, after chemical disequilibria are created ( $1^{\text {st }}$ Principle), entropy is produced by the available dissipative processes. But the same way water sometimes accumulates on its way downhill, kinetic barriers and/or unbalanced rates of dissipation may sustain large entropic dams (or free energy gradients) within the composition of a living system.

For long, the scientific community has wondered why nature chose phosphates (4). With the proposed principles, we may come up with a simplistic answer. At a pioneer stage of biological life, the formation of organophosphates was an accessible route for the dissipation of the created chemical disequilibria. Their characteristic double negative charge would have hindered their hydrolysis (39), which led to their accumulation and respective increase in chemical potential. Hence were born the entropic dams associated with phosphates, whose dissipation became highly favourable. With time, the exploration of that entropic dam became essential to the biological metabolism, enabling (i.e., constraining) many of its other processes. If organophosphates were no longer selected, biological life would stumble as it would lack an essential constraining component. Even if a new evolutionary succession restarted, this time without organophosphates, we would no longer recognise it as biological. That is the overarching reason why nature "chose" (4) phosphates.

The same reasoning applies to the formation and maintenance of significant out-of-equilibrium concentrations of various metabolites or chemiosmotic gradients. Remarkably, biological life has selected means to explore some of the entropic dams it creates. That is the basis for anabolism, catabolism, the heterotrophic lifestyle and the next sub-section.

The exploration of entropic dams. A living system requires environmental disequilibria to create chemical ones. To that purpose, chemical disequilibria (or entropic dams) within living systems provide similar opportunities.

As biological metabolisms evolved, they gained the ability to couple the dissipation of some of their entropic dams to the creation of other chemical disequilibria. Substrate-level phosphorylation and the conversion of redox gradients into chemiosmotic ones are well-known examples. Either labelled catabolism or anabolism, each biological metabolism is in fact a mesh of building systems (Fig. 1A). In fact, the same metabolism that by itself is a living system, a heterotroph, exists within any autotrophic organism. By extending this reasoning, we can understand that biological cells are communities of smaller metabolic living systems (Fig. 2). Consequently, it may be misleading to consider biological cells the basic unit of life as we know it.

\section{The properties of life}

Information. It has been accepted that living systems carry and inherit information. Only so would they have such a structured composition. To bring information under the umbrella of the present proposals, we 
may now recognise it to be the constraints exerted on the dissipative processes. More information (i.e., more constraints) implies a more structured composition.

In a living system, those constraints come from the environment and, according to the $2^{\text {nd }}$ Principle, from the living systems themselves (the self-constraints). So, part of the information comes from the environment and the rest of it from the living systems themselves. Thus, the information associated with any living system is the self-constraints it exerts on the dissipation of its chemical disequilibria.

That has been the intuition all along, with the information of a living system being commonly associated with its "genetic" subsystems. Indeed, they directly or indirectly constrain most of the composition of a living system, and by studying genetics we can predict biological phenotypes. But the same way other information inheritance mechanisms have been found (17), we can realise that any form of self-constraint is information. And that information is inherent to any living system ( $2^{\text {nd }}$ Principle), disregarding any need for dedicated genetic or any other informational subsystems (13).

Aliveness. To measure how much alive a living system is, we can use its inherent information - its selfconstraints. We can measure aliveness as the amount of information (i.e., constraints) a living system carries relative to the environment. We could assess it by how much of its composition is due to self-constraints relative to environmental ones. Aliveness would act as an indicator of the stage of evolution of a living system: the more information it carries relative to the environment, the more evolved it is.

A non-living building system (Fig. 1A) carries no self-constraints (no information); thus, it has no aliveness. A pioneer living system has a self-constrained dissipation, but its composition is still mostly determined by the environment; hence it has little aliveness (e.g., a community at early stages of succession, or the biological metabolism at its origin). On the contrary, the composition of a climax living system is essentially determined by the conditions it creates (its self-constraints), thus classifying at the top of the aliveness scale (e.g., a rainforest or a biological cell).

Quantifying selection. In life, selection regards the undertaking of dissipative routes. Through them, living systems produce entropy whilst dissipating previously created chemical disequilibria. Being so, we can quantify the selection of each dissipative route through its respective entropy production rate. It is equivalent to characterising the branching of a flow by measuring the flow rate in each direction. The higher the flow rate, the more selected that direction is.

In a more formal treatment, an out-of-equilibrium composition dissipates through different processes/routes $(X)$. We can quantify the selection of each one of them $\left(X^{j}\right)$ by measuring its entropy production rate $-\dot{S}^{j}$. We can thus quantify the selection of a dissipative process $\left(X^{j}\right)$ - or its respective product - relative to others by weighting such rate $-\dot{S}^{j} / \dot{S}^{x}$. 
Non-equilibrium thermodynamics provides us with the tools to compute the entropy production rate of chemical reactions and diffusion processes (40). When considering communities, we can quantify the selection of a climax living system (i.e., a species) as the rate at which entropy is being produced to form and maintain its specimens. We can then quantify its selection relative to alternatives (e.g., those with overlapping ecological niches) by comparing the rates of dissipation of the community disequilibria $-\dot{S}^{j} / \dot{S}^{x}$. If the species being compared have similar anabolic and catabolic efficiencies, we may approximate that entropy production ratio to a biomass production one $\dot{B}_{\text {prod }}^{j} /_{\dot{B}_{\text {prod }}^{X}}$. To this ratio - and the measurement of selection, overall - it is irrelevant whether the produced biomass implies more specimens, body growth, or metabolic maintenance.

Darwinian Evolution. Biological communities are living systems made of biological organisms. As the latter are in their later stage of evolution, they are self-replicating components of the former. The selection of these organisms implies increased production of their own biomass, which, most of the times, implies more copies of themselves.

Looking at the living components of biological communities, Darwin and others shortened the previous reasoning. They stipulated that the number of copies of a component (its abundance) was the absolute measure of its selection. Thus, they concluded, evolution shall favour higher reproductive success. It was simple and powerful at explaining the composition and evolution of the living species of macroscale communities. Because of that, Darwinian evolution was made a criterion for life (12).

Looking at it from the two principles, we understand the implicit limitations of the Darwinian approach (Fig. 3). First, the selection of a self-replicating component does not imply higher abundance. A mule is selected - from the dissipation of the chemical disequilibria stored in grasses - and more of its own biomass is produced. It creates more of itself, but not more mules. Thus, regarding the selection of self-replicating components, the production of their own biomass would provide a more accurate picture than abundance. Second, and most crucial, a component does not need to be self-replicating to be selected, i.e., to be a product of a dissipative route. Rate laws do not define which components of a living system are selected. It so happens that all living components of biological communities are self-replicating - because they are in their climax stages. But there is no need to extrapolate such a requirement.

As the selection of most biological organisms implies not only production of their own biomass but more copies of themselves, indeed their selection goes hand-in-hand with reproductive success ("fitness"). And considering similar body masses, we can estimate their relative selection through their relative abundances. So, "natural selection" can be a proxy for actual selection in such conditions, to which Darwinian evolution has proven to be a valuable model (Fig. 3). But it is certainly not a criterion for the phenomenon of life. 


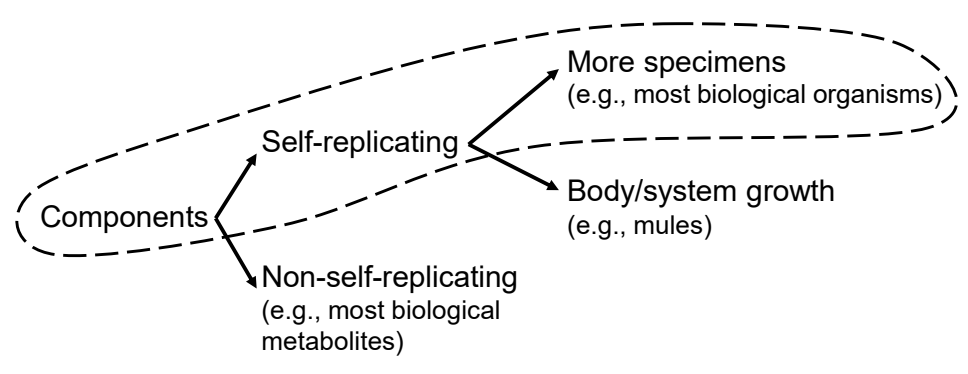

Fig. 3. The domain of Darwinian evolution. Natural selection can be a proxy for actual selection when considering self-replicating components whose selection implies more copies of themselves (dashed boundary).

\section{The origin of life}

Which pioneer organisms originated the Amazon rainforest? That question is entirely analogous to the description of the origin of the biological metabolism. And it does not become less daunting by analysing extant metabolisms. That is as useful as studying the extant Amazon to understand its origin. We should study the origin of life by attempting to reproduce it, rather than extrapolating from biological cells. Therefore, in this last section, I deduce an ab initio scenario for the emergence of biological life and leave the description of pioneer compositions for future experimental work.

The creation of chemical disequilibria. Although some metabolisms evolved means of coupling sunlight to the creation of chemiosmotic gradients (41), virtually all biological life starts from an excited (i.e., outof-equilibrium) distribution of electrons. And it was never different. The same way a living community collapses if it loses its autotrophic organisms, it is nearly inconceivable that a living metabolism would not stumble if its original chemical disequilibrium was no longer created. A living system which loses its autotrophic structure has its information (its self-constraints) turned senseless. Therefore, we can assume that an evolutionary succession with electrons being persistently excited took biological life from its origin to the extant forms.

We may thus conclude that at the hatchery of biological life there must have been a persistent creation of out-of-equilibrium electron distributions. Nowadays, such chemical disequilibria are created from sunlight or redox gradients. They then dissipate towards identical metabolisms, independently of which environmental disequilibrium is explored. That indicates that the biological metabolism evolved its extant shape whilst exploring one disequilibrium, and only then it evolved the means to explore the second one. That also implies that before them, biological life may have explored yet a third, unknown, environmental disequilibrium. Let us assume it did not.

Although sunlight readily creates electronic excited states, it seems unlikely for such chemical disequilibria to dissipate orderly and periodically enough to meet the $2^{\text {nd }}$ Principle; besides the obstacles brought by the toxicity of ultraviolet radiation to organic molecules. In contrast, it is much easier to find redox gradients 
in more stable and less toxic conditions. Conditions where we can imagine the $2^{\text {nd }}$ Principle being met. But is it plausible that the dissipation of a redox gradient would spontaneously take electrons out-ofequilibrium?

Electron bifurcation. By themselves, two chemical processes resolve independently (27). For an electron to be excited whilst a redox gradient is dissipated, some mechanism must couple the processes. In biological systems, there is only one mechanism capable of it: electron bifurcation (42).

Electron bifurcation is based on the two-electron oxidation of a molecular species with inverted potentials $(43,44)$. In such species, after a single-electron exergonic oxidation, a second electron goes up in oxidation potential (Fig. 4). This way, part of the disequilibrium that drives the first oxidation is preserved in an increased disequilibrium associated with the second oxidation. An evidence of it is that the second oxidation reduces species with higher oxidation potentials than was previously possible - interpreted as an endergonic reaction (42). Thus, through this mechanism widespread across extant living systems (45), the dissipation of one redox gradient (the first oxidation) creates another chemical disequilibrium ( $1^{\text {st }}$ Principle).

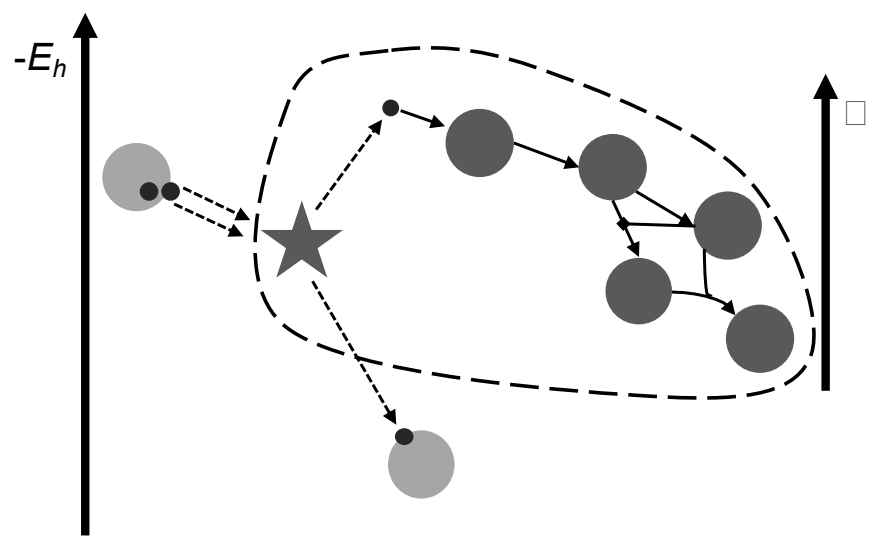

Fig. 4. Schematic representation of electron bifurcation and how it fulfils the $1^{\text {st }}$ Principle of life. From the two electrons (small dark grey) transferred from an environmental (light grey) electron donor to a bifurcating centre (star), one proceeds to an environmental acceptor. The bifurcating centre converts part of the free energy of that exergonic redox reaction to an increased chemical disequilibrium associated with the second oxidation. Together with a self-constraining dissipation, electron bifurcation may originate a living system (enclosed by dashed boundary).

About 45 years ago, Peter Mitchell predicted that electron bifurcation operates in the Q-cycle of the mitochondrial Electron Transport Chain using quinones as bifurcating centres (46). While studying anaerobic bacteria (47), researchers have concluded that other two-electron redox molecules enable this mechanism. They have discovered several instances of flavin-based electron bifurcation (45) and proposed that $\mathrm{Mo} / \mathrm{W}$-pterin centres also promote this mechanism (48). 
The beginning of life on Earth. Electron bifurcation (or potential inversion) is an appealing coupling (or free energy converting) mechanism to the origin of life because of its general physicochemical nature (43), being accessible to wide-ranging molecules under different conditions (44). And indeed, researchers have shown that certain molybdenum and tungsten organometallic complexes attain inverted potentials $(49,50)$. This goes to show how the right geological landscape and suitable redox gradients could have serendipitously created chemical disequilibria in the early stages of Earth.

For different reasons, experimental evidence has been mounting towards hydrothermal vents as the hatchery of life $(36,51,52)$. In the light of the present discussion, the maintenance of high concentrations of molecular hydrogen - which would act as the environmental two-electron donor (Fig. 4) - and the high abundance of transition metals - some of which could attain inverted potentials - confirm hydrothermal sites as the most probable hatcheries of life as we know it (53).

Regarding the fixation of carbon, the proton-coupled two-electron reduction of carbon dioxide is far more favourable than the reduction with a single electron (54). In particular, the acetyl coenzyme A pathway for carbon fixation requires the reduction of $\mathrm{Ni}^{+/ 2+}$ species to metallic nickel $\left(\mathrm{Ni}^{0}\right)$ before reducing carbon dioxide (55). To become organic, biological life may have required an analogous scheme at its hatchery.

Subsequent exergonic and endergonic (through electron bifurcation) reactions led to more complex molecules and to the fixation of other elements. As the metabolites reacted with one another; as hydroxyl and carboxyl groups buffered the $\mathrm{pH}$ of the solution; as new organometallic compounds catalysed some chemical reactions; or as autocatalytic metabolites were formed, the dissipation of the chemical disequilibria became self-constrained. With that, the evolution of biological life smoothly started.

Colonising the world. After an evolutionary succession in a hydrothermal vent, and the selection of amphiphiles and electron bifurcating components, biological life became cellular and ready to colonise other habitats. Deep-ocean currents and/or tectonic events could have carried biological metabolisms to suitable hydrothermal environments. One of which may have been in the euphotic zone, such as shallow hydrothermal vents (56). Then, in the overlap of the two disequilibria, biological metabolisms could have evolved the means to convert sunlight into chemical disequilibria. As both photosynthesis and electron bifurcation create chemical disequilibria as excited electrons, the information on their dissipation was still relevant. The metabolism evolved until then could be preserved. Hence the similarity between the biological metabolisms at the bottom of the ocean and those at the surface.

The wandering chemoautotrophic branch of life would have no reason to evolve from its hydrothermaladapted climax metabolisms. It would thus be fair to consider them the most ancient metabolisms, from which the photoautotrophic branch of life evolved. Fortunately, phylogenetic studies have endorsed this hypothesis by indicating that the most deep-branching organisms live in extant hydrothermal vents (57). 


\section{Final Remarks}

Life is a natural (12) phenomenon that the scientific community has struggled to put into simple principles. Starting from biased observations, it created prejudiced models of life. Given their imprecision, we could not recreate life. And the lack of new experimental evidence hindered the improvement of our models.

To break that vicious cycle, I have proposed life to be the creation of chemical disequilibria and their selfconstrained dissipation. Therefore, a living system is the process of creation of chemical disequilibria and all their interacting dissipative processes. Selection has been reinterpreted as the undertaking of those dissipative processes, and evolution as a change in the constraints on them. It was then shown how the principles of life explain the biological composition and the established pillars of life.

If those are the universal principles of life, we can expect non-living matter to transition into a living system every time we replicate them experimentally. In particular, by replicating the electron bifurcation mechanism within proper environmental constraints, we may now be underway to replicate the origin of life on Earth. If successful, it will be the beginning of a new scientific field of living chemistry, intended to create, explore and model living systems. With that knowledge, we shall, at last, understand the origin of biological life and address its related questions: Which mechanisms constrained the formation of the genetic code and associated machinery? How long did it take for the Last Universal Common Ancestor (LUCA) to emerge? What did LUCA look like?

The primary goal of this work was to lay the foundations for the ultimate understanding of life. As we experiment with them, we will fine tune our search for alternative life forms with varying degrees of aliveness. By now, we can already state that habitability criteria should include not only free energy sources (58) but also their transduction into chemical disequilibria. How different environmental disequilibria may be converted into chemical ones is an exciting subject for future experimental work, which will inform the possibility of life in different extra-terrestrial habitats. The results of those enquires will spawn a technology whose potential is nothing shorter than the whole biosphere and everything it has ever built.

\section{References}

1. Merindol R, Walther A. Materials learning from life: Concepts for active, adaptive and autonomous molecular systems. Chemical Society Reviews. 2017;46(18):5588-619.

2. Ashkenasy G, Hermans TM, Otto S, Taylor AF. Systems chemistry. Chemical Society Reviews. 2017;46(9):2543-54.

3. Preiner M, Asche S, Becker S, Betts HC, Boniface A, Camprubi E, et al. The future of origin of life research: Bridging decades-old divisions. Life. 2020 Feb 26;10(3).

4. Westheimer FH. Why nature chose phosphates. Science. 1987 Mar;235(4793):1173-8.

5. Koonin E V., Novozhilov AS. Origin and Evolution of the Universal Genetic Code. Annual Review of Genetics. 2017;51:45-62. 
6. Smith E, Morowitz HJ. Universality in intermediary metabolism. Proceedings of the National Academy of Sciences of the United States of America. 2004;101(36):13168-73.

7. Benner SA, Ricardo A, Carrigan MA. Is there a common chemical model for life in the universe? Current Opinion in Chemical Biology. 2004;8(6):672-89.

8. Schwieterman EW, Kiang NY, Parenteau MN, Harman CE, Dassarma S, Fisher TM, et al. Exoplanet Biosignatures: A Review of Remotely Detectable Signs of Life. Astrobiology. 2018 Jun 1;18(6):663-708.

9. Benner SA. Defining life. Astrobiology. 2010 Dec;10(10):1021-30.

10. Weber BH. What is life? defining life in the context of emergent complexity. Origins of Life and Evolution of Biospheres. 2010;40(2):221-9.

11. Luisi PL. About various definitions of life. Origins of Life and Evolution of the Biosphere. 1998;28(4-6):613-22.

12. Cleland CE, Chyba CF. Defining "life." Origins of Life and Evolution of the Biosphere. 2002;32(4):387-93.

13. Koshland DE. The seven pillars of life. Science. 2002;295(5563):2215-6.

14. Benner SA, Hurter D. Phosphates, DNA, and the search for nonterrean life: A second generation model for genetic molecules. Bioorganic Chemistry. 2002;30(1):62-80.

15. Varela FG, Maturana HR, Uribe R. Autopoiesis: The organization of living systems, its characterization and a model. BioSystems. 1974;5(4):187-96.

16. Chodasewicz K. Evolution, reproduction and definition of life. Theory in Biosciences. 2014;133(1):39-45.

17. Danchin É, Charmantier A, Champagne FA, Mesoudi A, Pujol B, Blanchet S. Beyond DNA: Integrating inclusive inheritance into an extended theory of evolution. Nature Reviews Genetics. 2011;12(7):475-86.

18. Wächtershäuser G. From volcanic origins of chemoautotrophic life to Bacteria, Archaea and Eukarya. Philosophical Transactions of the Royal Society B: Biological Sciences. 2006;361(1474):1787-806.

19. Weber AL. The sugar model: Autocatalytic activity of the triose-ammonia reaction. Origins of Life and Evolution of the Biosphere. 2007;37(2):105-11.

20. Joyce GF. The antiquity of RNA-based evolution. Nature. 2002;418(6894):214-21.

21. Deamer D. The role of lipid membranes in life's origin. Life. 2017;7(1).

22. Muchowska KB, Varma SJ, Moran J. Nonenzymatic Metabolic Reactions and Life's Origins. Chemical Reviews. 2020;120(15):7708-44.

23. Blackmond DG. An Examination of the Role of Autocatalytic Cycles in the Chemistry of Proposed Primordial Reactions. Angewandte Chemie International Edition. 2009;48(2):386-90. 
24. Orgel LE. The implausibility of metabolic cycles on the prebiotic earth. PLoS Biology. 2008;6(1):5-13.

25. Sutherland JD. Opinion: Studies on the origin of life - The end of the beginning. Nature Reviews Chemistry. 2017;1.

26. Walker SI. Origins of life: A problem for physics, a key issues review. Reports on Progress in Physics. 2017 Aug 14;80(9):092601.

27. Kostic MM. The Second Law and Entropy Misconceptions Demystified. Entropy. 2020;22(6).

28. Kaufmann M. On the free energy that drove primordial anabolism. International Journal of Molecular Sciences. 2009;10(4):1853-71.

29. Branscomb E, Russell MJ. Turnstiles and bifurcators: the disequilibrium converting engines that put metabolism on the road. Biochimica et biophysica acta. 2013 Feb;1827(2):62-78.

30. Miller SL. A production of amino acids under possible primitive earth conditions. Science. 1953;117(3046):528-9.

31. Cable ML, Hörst SM, Hodyss R, Beauchamp PM, Smith MA, Willis PA. Titan tholins: Simulating Titan organic chemistry in the Cassini-Huygens era. Chemical Reviews. 2012;112(3):1882-909.

32. Epstein IR. Coupled chemical oscillators and emergent system properties. Chemical Communications. 2014;50(74):10758-67.

33. Trifonov EN. Vocabulary of Definitions of Life Suggests a Definition. Journal of Biomolecular Structure and Dynamics. 2011 Oct 1;29(2):259-66.

34. Ricklefs RE. The Economy of Nature. 6th ed. New York, NY: W. H. Freeman \& Co.; 2008.

35. Van Zuilen MA, Lepland A, Arrhenius G. Reassessing the evidence for the earliest traces of life. Nature. 2002 Aug 8;418(6898):627-30.

36. Djokic T, Van Kranendonk MJ, Campbell KA, Walter MR, Ward CR. Earliest signs of life on land preserved in ca. 3.5 Ga hot spring deposits. Nature Communications. 2017;8(1):15263.

37. Wolos A, Roszak R, Zadlo-Dobrowolska A, Beker W, Mikulak-Klucznik B, Spólnik G, et al. Synthetic connectivity, emergence, and self-regeneration in the network of prebiotic chemistry. Science. 2020;369(6511).

38. Bludau I, Aebersold R. Proteomic and interactomic insights into the molecular basis of cell functional diversity. Nature Reviews Molecular Cell Biology. 2020 Jun 1;21(6):327-40.

39. Keefe AD, Miller SL. Are polyphosphates or phosphate esters prebiotic reagents? Journal of Molecular Evolution. 1995;41(6):693-702.

40. Kondepudi D, Prigogine I. Nonequilibrium Thermodynamics: The Foundations. In: Modern Thermodynamics: From Heat Engines to Dissipative Structures. 2nd ed. 2014. p. 341-56. (Wiley Online Books).

41. Luecke H, Schobert B, Richter HT, Cartailler JP, Lanyi JK. Structural changes in bacteriorhodopsin during ion transport at 2 angstrom resolution. Science. 1999 Oct 
$8 ; 286(5438): 255-60$.

42. Müller V, Chowdhury NP, Basen M. Electron Bifurcation: A Long-Hidden Energy-Coupling Mechanism. Annual review of microbiology. 2018 Sep;72:331-53.

43. Nitschke W, Russell MJ. Redox bifurcations: Mechanisms and importance to life now, and at its origin: A widespread means of energy conversion in biology unfolds... BioEssays.

2012;34(2):106-9.

44. Evans DH. One-electron and two-electron transfers in electrochemistry and homogeneous solution reactions. Chemical Reviews. 2008 Jul;108(7):2113-44.

45. Buckel W, Thauer RK. Flavin-Based Electron Bifurcation, A New Mechanism of Biological Energy Coupling. Chemical Reviews. 2018 Apr 11;118(7):3862-86.

46. Mitchell P. The protonmotive Q cycle: A general formulation. FEBS Letters. 1975 Nov 15;59(2):137-9.

47. Herrmann G, Jayamani E, Mai G, Buckel W. Energy conservation via electron-transferring flavoprotein in anaerobic bacteria. Journal of Bacteriology. 2008 Feb 1;190(3):784-91.

48. Baymann F, Schoepp-Cothenet B, Duval S, Guiral M, Brugna M, Baffert C, et al. On the natural history of flavin-based electron bifurcation. Frontiers in Microbiology. 2018;9(JUL).

49. Uhrhammer D, Schultz FA. Energetics of concerted two-electron transfer and metal-metal bond cleavage in phosphido-bridged molybdenum and tungsten carbonyl complexes. Journal of Physical Chemistry A. 2002 Nov 28;106(47):11630-6.

50. Muratsugu S, Sodeyama K, Kitamura F, Tsukada S, Tada M, Tsuneyuki S, et al. Normal and inverted redox potentials and structural changes tuned by medium effects in [M2Mo( $75-$ C5Me5)2(S2C6H4)2(CO)2] (M: Co, Rh). Chemical Science. 2011 Oct 5;2(10):1960-8.

51. Dodd MS, Papineau D, Grenne T, Slack JF, Rittner M, Pirajno F, et al. Evidence for early life in Earth's oldest hydrothermal vent precipitates. Nature. 2017 Mar 1;543(7643):60-4.

52. Javaux EJ. Challenges in evidencing the earliest traces of life. Nature. 2019;572(7770):451-60.

53. Russell MJ, Barge LM, Bhartia R, Bocanegra D, Bracher PJ, Branscomb E, et al. The drive to life on wet and Icy Worlds. Astrobiology. 2014 Apr 1;14(4):308-43.

54. Benson EE, Kubiak CP, Sathrum AJ, Smieja JM. Electrocatalytic and homogeneous approaches to conversion of CO2 to liquid fuels. Chemical Society Reviews. 2009;38(1):89-99.

55. Fuchs G. Alternative pathways of carbon dioxide fixation: Insights into the early evolution of life? Annual Review of Microbiology. 2011;65:631-58.

56. Nisbet EG, Sleep NH. The habitat and nature of early life. Nature. 2001;409(6823):1083-91.

57. Weiss MC, Sousa FL, Mrnjavac N, Neukirchen S, Roettger M, Nelson-Sathi S, et al. The physiology and habitat of the last universal common ancestor. Nature Microbiology. 2016;1(9).

58. Cockell CS, Bush T, Bryce C, Direito S, Fox-Powell M, Harrison JP, et al. Habitability: A Review. Astrobiology. 2016 Jan 1;16(1):89-117. 